\title{
La gestion des talents au sein du secteur bancaire marocain
}

\author{
Wafa DRIOUA \\ Docteur en sciences de gestion, Université Montpellier 3, Montpellier, France
}

\begin{abstract}
This article examines the evolution of human resources management in the Moroccan banking sector concerning the advent of a new approach "Talent". This sector, which is characterized by violent competition between its various establishments, is currently experiencing an exceptional rise. It is considered one of the most structured and performing sectors in Morocco. Our main objective is to see if talent management can be imposed in Moroccan banks as a new style to human resources management. The work that has examined the notion of talent since it appeared in management sciences it just been clarified a little of this concept. Thus, the notion of talent always raises questions and provokes debates. We elected for the multiple case study method. Our case study focuses on the largest three Moroccan banks classified first nationally. As a result, we have chosen to carry out a qualitative survey of the employees of these three banks. More specifically, we propose to observe, in the first instance, the current policy realized by these banks in terms of human resources management. In a second phase, we intend to shed light on the emergence of the new approach "Talent", and to also discern its place within these three banks.
\end{abstract}

Keywords: Human resources management - talent - Moroccan banking sector - human resources policy competition.

Résumé: Cet article a pour objet l'étude de l'évolution de la gestion des ressources humaines au sein du secteur bancaire marocain vers l'avènement d'une nouvelle approche "Talents 》. Ce secteur qui est caractérisé par une concurrence acharnée entre ses différents établissements, connaît actuellement un essor sans précédent. Il est considéré comme l'un des secteurs les plus structurés et les plus performants au Maroc. Notre objectif principal consiste à voir si la gestion des talents peut s'imposer au sein des banques marocaines comme une nouvelle approche de la gestion des ressources humaines. Les travaux qui se sont penchés sur la notion du talent depuis qu'elle a fait son émergence en sciences de gestion n'ont, en effet, que peu permis de clarifier ce concept. Ainsi, la notion du talent soulève toujours des questionnements et provoque des débats. Nous avons opté pour la méthode de l'étude de cas multiples. Notre étude de cas porte sur les trois plus importantes banques marocaines classées premières à l'échelle nationale. Pour cela, nous avons choisi de réaliser une enquête qualitative auprès des salariés de ces trois banques. Plus particulièrement, nous proposons d'observer, dans un premier temps, la politique actuelle mise en place par ces banques en matière de gestion des ressources humaines. Dans un second temps, nous envisageons d'apporter un éclairage sur l'apparition de la nouvelle approche «Talents », et à discerner également sa place au sein de ces trois banques.

Mots-clés: Gestion des ressources humaines-talent- secteur bancaire marocain - politique de ressources humaines-concurrence.

\section{Introduction}

La Gestion des Ressources Humaines (GRH) revêt une importance cruciale durant notamment ces dix dernières années. Elle a évolué au fil du temps, c'est le résultat d'un long processus de la gestion du travail ; elle se trouve aujourd'hui au cœur de la stratégie de l'entreprise. Ce changement dans la GRH est dû essentiellement aux différents changements qui se sont opérés dans les organisations elles-mêmes. Il ne fait plus de doute que l'entreprise a besoin aujourd'hui non seulement des personnes qui accomplissent les missions et les tâches définies, mais elle a aussi besoin des personnes porteuses de connaissance, et source d'innovation, car ce qui fait la richesse de l'entreprise sont ses Ressources Humaines (RH), elles sont les véritables ressources génératrices de richesses. Ainsi, les RH sont considérées comme un facteur clé de la performance des entreprises.

Désormais, on ne parle plus de compétence mais plutôt de talent. La notion de talent est souvent utilisée dans un contexte sportif ou artistique, il s'agit de décrire des personnes dotées de performances remarquables. Elle a été pratiquement absente de la littérature consacrée à la gestion. Or, depuis quelques années, cette terminologie a été utilisée par les auteurs et les praticiens de sciences de gestion.

L'avènement de la nouvelle approche $\mathrm{RH}$ « Talents » a suscité notre intérêt, il s'agit de s'intéresser à la nouveauté qu'elle introduit et qui justifie l'utilisation de cette nouvelle notion dans les sciences de gestion. L'objet de notre recherche consiste à comprendre si la gestion des talents peut s'imposer au sein des banques marocaines comme une nouvelle approche RH. En effet, ce thème reste peu exploré dans le cadre des recherches en sciences de gestion au Maroc. De même, il y a peu de recherches empiriques traitant ce sujet. À notre connaissance, notre recherche constitue l'une des premières au Maroc. 
Le Maroc, terrain de notre travail de recherche, est un pays qui a commencé à changer de visage lors des dernières années, on parle du " Maroc qui bouge », il est désormais tourné vers l'avenir. L'histoire de la GRH au Maroc est récente, elle est apparue au début du siècle dernier avec la colonisation et l'introduction du capitalisme. Aujourd'hui, les dirigeants, les théoriciens, les chercheurs, et les praticiens marocains accordent une attention particulière à la GRH. Dans ce contexte, plusieurs initiatives ont été prises et plusieurs formations sont lancées dans ce sens, afin de construire un modèle GRH, et de moderniser ses outils et ses pratiques. Ce pays vit depuis une dizaine d'années au rythme de profonds changements et de grandes mutations d'ordre politique, économique, social...etc.

Le champ de notre recherche concerne le secteur bancaire marocain, l'un des secteurs les mieux structurés au Maroc. Ce secteur est aujourd'hui le plus recherché des jeunes. Il est l'un des premiers employeurs privés du Maroc. Son attractivité est due notamment au métier de banquier. Celui-ci est reconnu comme étant un métier prestigieux, et qui offre des perspectives d'évolution de carrière intéressantes. En effet, en intégrant un secteur qui est considéré comme l'un des secteurs les mieux structurés au Maroc, ces jeunes dynamiques cherchent l'enrichissement et la qualité de l'expérience professionnelle que ce secteur peut leur offrir. De même, le secteur bancaire marocain compte des parcours professionnels intéressants avec une particularité fondamentale représentée par la mutualisation de la formation dans le secteur.

Il convient de souligner que le secteur bancaire privilège davantage les jeunes diplômés dans la perspective de «dynamiser les équipes et rajeunir leur image de marque». Aussi, compte tenu de la nature de son travail basé essentiellement sur la réalisation des objectifs et des chiffres, les profils commerciaux et les métiers de la force de vente en général sont les plus recherchés par ce secteur. La quête des jeunes talentueux caractérisés par une agressivité commerciale remarquable est donc une priorité primordiale du secteur.

Il faut savoir qu'au sein de ce secteur tout est basé sur les chiffres, cela veut dire que la compétence du salarié et sa performance se mesurent à travers ses réalisations individuelles en termes de chiffres et d'objectifs. Le secteur bancaire exige aussi de la part de ses salariés la polyvalence. En effet, il ne suffit plus de faire preuve d'expertise uniquement dans le cadre de sa fonction. Aujourd'hui, les banquiers sont amenés à faire preuve d'une plus grande polyvalence.

Par ailleurs, suite à la crise actuelle que connait le marché du travail, les candidats sont plus nombreux sur ce marché, ce qui rend la concurrence plus forte. Ce constat nous laisse penser que face à la forte demande, les banques ont plus de choix quant aux critères de sélection de leurs futurs salariés. En fait, elles exigent de plus en plus des profils de grande compétence, et leur politique RH est orientée aujourd'hui plus que jamais aux jeunes cadres.

Le secteur bancaire marocain que nous avons choisi pour notre étude empirique, illustre parfaitement notre problématique, en raison de la concurrence acharnée entre les différents établissements bancaires en termes de RH, et également de l'effectif employé. Notre objectif consiste à repérer la place de la nouvelle approche RH «Talents » au sein des banques marocaines. Pour cela, et afin d'avoir une vision plus claire à ce sujet, nous allons tenter de répondre à cette question clé :

Les banques marocaines adoptent-elles la nouvelle approche RH « Talents »?

\subsection{L'apparition de la notion de talent}

\section{Le talent : une nouvelle approche RH}

L'apparition d'une réflexion théorique sur cette notion a eu lieu dans les années 2000. En effet, plusieurs phénomènes ont été à l'origine de son apparition. Elle est née dans un contexte d'hyper compétition, de pénurie de la main d'œuvre, de manque massif de compétences, et de désengagement de l'employé.

La notion de talent a fait son apparition dans l'univers managérial pour la première fois en 2000, dans une étude menée par le cabinet Mc Kinsey. Il s'agit d'un cabinet anglo-saxon spécialiste des problématiques de RH. C'est l'un des plus grands cabinets mondiaux de conseil auprès des directions générales. A ce propos, Cadin (2009) [1] souligne la richesse de cette terminologie choisie par ce cabinet : une référence directe au monde du sport et au monde des arts, mais aussi à l'univers des artisans (Dejoux et Thévenet, 2010) [2] qui a inspiré fortement les entreprises.

Ainsi, cette nouvelle notion du talent, est apparue dans les travaux des auteurs du célèbre ouvrage " The war for talents», écrit par des consultants de Mc Kinsey (Michaels, Handfield-Jones et Axelrod) [3], et à la suite duquel un courant de pensée d'origine américaine, appelé Talent Management « Management des talents » a fait son apparition en 2001, dont la démarche est centrée sur le talent.

En revanche, peu de travaux se sont penchés sur la question du talent, ce sujet reste peu abordé dans les recherches en France et encore moins au Maroc.

Par ailleurs, il existe encore des problèmes au niveau de la façon dont le talent management a été défini par les praticiens, et il y a un manque de données à l'appui de nombreuses revendications de ces derniers. Lewis et Heckman (2006) [4] soulignent le manque de clarification théorique de cette notion. Actuellement, le débat reste ouvert, car même la définition de cette notion ne fait pas l'unanimité auprès des chercheurs. 


\subsection{Qu'est-ce que le talent ?}

Selon divers dictionnaires (Andrieux-Reix, 2004; Rey, 2010), l'origine étymologique du mot talent remonte à la Grèce antique où le talent référait à une mesure de poids. Il représentait des valeurs différentes selon son métal, et selon compte les régions de l'Empire, il devient ainsi une notion monétaire.

En ancien français et jusqu'au XVIe siècle, après avoir eu le sens d'« humeur » et « état d'esprit », le talent a signifié « désir », « volonté », sens que l'on retrouve dans les locutions avoir en talent (« désirer »), faire son talent de («agir à son gré »), dire son talent («donner son avis »).

Comme l'indiquent les dictionnaires « est talentueux », « celui qui a des aptitudes remarquables pour réussir en quelque chose », «celui qui a du talent » ou même celui « qui a beaucoup de talent ».

Le Robert définit le talent comme « une disposition, naturelle ou acquise, pour réussir en quelque chose » ou comme « une aptitude remarquable dans le domaine intellectuel ou artistique », ou comme "le don littéraire ou artistique (de quelqu'un) considéré dans ses caractères propres ». Le terme talent a trois significations selon le Robert : don, aptitude «pour réussir »; art, capacité, génie, faculté, instinct, qualité ; « aptitude particulière, dans une activité appréciée par le groupe social ».

Selon le Larousse (2013), un talent est une aptitude, une capacité particulière à faire quelque chose. Il désigne aussi « un don remarquable dans le domaine artistique, littéraire ; une personne douée en telle activité ».

Le Larousse et le Robert définissent le talent en utilisant des termes comme aptitude, capacité remarquable ou particulière et don. Le talent est toujours lié à une activité en particulier et sa source/origine fait référence à de l'inné ou de l'acquis. En emploi absolu, le talent se dit d'une aptitude remarquable dans le domaine intellectuel ou artistique.

Mirallès (2007) [5] définit le talent comme « excellence plus différence : une excellence individuelle dans une activité donnée. Pour lui, si la compétence d'un individu se définit par ce qu'il sait faire, son talent est caractéristique de ce qu'il fait mieux que les autres.

Pour notre part, la notion du talent correspond à la capacité d'un individu à apporter une valeur additionnelle, et à se distinguer des autres en disposant d'un potentiel et des compétences spécifiques. Cela implique sa capacité à faire mieux et plus vite que les autres, en ayant la volonté d'exceller dans son activité professionnelle.

Nous définissons le talent comme "une combinaison des compétences hors du commun " (Drioua, 2014). Ainsi, le talent peut être défini non seulement comme une personne motivée, et compétente, qui assure le bon fonctionnement du travail; mais une personne talentueuse, c'est bien une personne qui révèle une compétence hors du commun. Elle détient une combinaison de compétences rares.

Il convient de souligner qu'à ce jour la notion de talent ne possède pas de définition communément admise, et par conséquent, il n’y pas de consensus sur la définition du talent. En effet, certains chercheurs considèrent les talents comme des personnes clés, dotées d'un haut potentiel et d'une performance exceptionnelle leur permettant de se différencier des autres. Ce qui veut dire que le talent se limite à une minorité de personnes. Il s'agit d'une catégorie de personnel pouvant accéder à des postes de responsabilité dans un court terme. Tandis que d'autres, considèrent que le talent se trouve au cœur de chaque personne, c'està-dire chacun en est doté, en attendant qu'il soit découvert.

Selon Chaminade (2003) [6], le talent ne se limite pas aux leaders ou bien aux cadres. D'après lui, le talent désigne « toute personne, dont les capacités représentent une plus-value pour l'organisation, en lui permettant de se différencier de ses concurrents ».

\subsection{La gestion des talents : des nouvelles pratiques $\mathbf{R H}$}

Pour que les entreprises assurent leur compétitivité, il ne suffit pas seulement de disposer des talents. En effet, la notion de talent fait référence au développement des nouvelles pratiques RH. Ainsi, le management des talents, est défini comme « l'attraction systématique, l'identification, le développement, la fidélisation et le déploiement des individus avec un haut potentiel qui ont une valeur particulière pour l'organisation » (Chartered Institute of Personnel and Development, 2006).

Cela nous renvoie aux nouvelles pratiques du management des talents. A cet effet, certains auteurs considèrent le management des talents comme une approche RH qui fait référence à de nouvelles pratiques bien déterminées. Tandis que d'autres, la renvoie à de simples pratiques de gestion prévisionnelle.

D'après la figure suivante qui définit le management des talents selon De BRY F. et GOURDON C. (2009) [7], il découle que le management des talents supposent d'implanter, identifier, mobiliser, développer, et régénérer les talents. 
Figure 1. Le management des talents

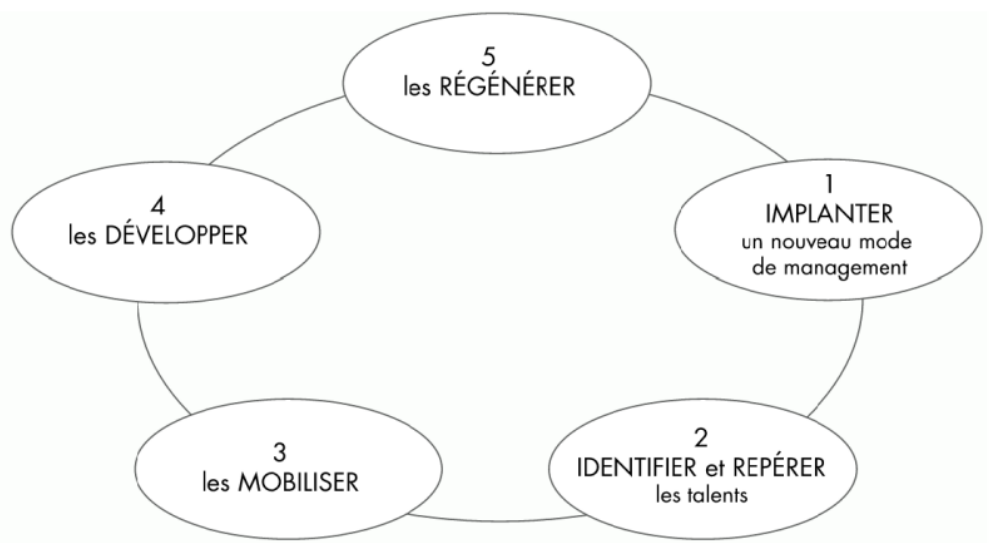

Source : De BRY F. et GOURDON C. (2009)

Quant à Mirallès (2007, p.161), il formule trois pratiques du management des talents en faisant un parallèle avec le football, il s'agit de :

$1 /$ «Reconnaître les talents » : savoir les détecter (scouting);

$2 /$ «Exploiter les talents » : créer les meilleures conditions de leur expression spécifique (coaching) et savoir les combiner entre eux pour tirer le meilleur parti de leurs complémentarités (casting);

$3 /$ «Protéger les talents » : les isoler des tentations externes (cocooning).

De leur côté, Dejoux et Thevenet (2010) décomposent la gestion avec les talents en trois phases :

1/ Attirer les talents ;

2/ Recruter les talents ;

3/ Fidéliser les talents.

Pour notre part, afin de traiter la question des pratiques de la gestion des talents, nous estimons que le management des talents apporte trois nouvelles pratiques, à savoir : attirer les talents, accompagner les talents, et fidéliser les talents. Nous résumons ces pratiques dans la figure suivante :

Figure 2. Les nouvelles pratiques du management des talents

\begin{tabular}{|c|}
\hline Attirer les talents \\
\hline $\begin{array}{l}\text { - Construire sa marque employeur } \\
\text { - Définir et positionner son offre employeur } \\
\text { - Mettre en place des méthodes de recrutement innovantes } \\
\text { - Identifier les talents en inteme } \\
\text { - Dénicher les talents extemes }\end{array}$ \\
\hline Accompagner les talents \\
\hline $\begin{array}{l}\text { - Mettre en place un parcours d'intégration } \\
\text { - Adhérer les talents à la culture d'entreprise } \\
\text { - Développer les talents } \\
\text { - Mangement de proximité } \\
\text { - Coaching }\end{array}$ \\
\hline Fidéliser les talents \\
\hline $\begin{array}{l}\text { - Offrir des conditions de travail favorables } \\
\text { - Impliquer et motiver les talents } \\
\text { - Développer le sentiment d'appartenance } \\
\text { - Reconnaitre et valoriser les talents }\end{array}$ \\
\hline
\end{tabular}

Source : Elaborée par l'auteur

III. Gestion des talents et performance des entreprises : quelle relation ?

3.1 Définition du concept de la performance

En présence d'une concurrence acharnée, les entreprises aujourd'hui sont de plus en plus à la recherche d'un avantage concurrentiel durable leur permettant de se distinguer par rapport aux concurrents. Ainsi, on peut juger une entreprise performante, quand elle est capable de maintenir son avantage concurrentiel durable, et d'assurer sa pérennité par une création permanente de valeur. 
Le mot performance provient du verbe anglais « to perform », et qui a fait son apparition au 14ème siècle. C'est un verbe qui est issu du vieux français " parformer ", qui au 13ème siècle signifiait " accomplir, exécuter ». En effet, la définition du concept de performance a suscité l'intérêt de plusieurs chercheurs, plus particulièrement depuis les années 80 (Bouquin, 1986 ; Bescos et al.1993 ; Bourguignon, 1995 ; Lebas, 1995 ; Bessire, 1999) [8]. Selon Bourguignon (1999), la performance est définie comme « la réalisation des objectifs organisationnels, quelle que soit la nature et la variété de ces objectifs. Cette réalisation peut se comprendre au sens strict (résultat, aboutissement) ou au sens large du processus qui mène au résultat (action) » (Bourguignon, 1997, p.934). Ce qui veut dire que l'interprétation de la réalisation peut se faire au sens large en ce qui concerne le processus qui mène au résultat «action ». Comme elle peut être interprétée au sens strict "résultat, aboutissement ». Il convient de dire que cette définition s'applique autant à l'organisation qu'à l'individu : « est performant celui ou celle qui atteint ses objectifs » (Bourguignon, 1995, p.65) [9].

Il est à souligner qu'il n'y pas de consensus sur la définition du concept de la performance. Celle-ci a toujours été une notion ambiguë dans le cadre des sciences de gestion. Elle s'assimile traditionnellement, à la réalisation et à l'atteinte des objectifs de l'organisation, voire même leur dépassement.

\subsection{Les talents comme facteur clé de la performance}

À partir de 1985, de nombreux travaux de recherche se sont penchés sur la question de la performance et la GRH. On constate donc l'apparition de plusieurs travaux qui traitent le lien de causalité pouvant exister entre la GRH et la performance. Ces divers travaux, tentent d'expliquer que la compétence, son acquisition, son développement, sa gestion, s'imposent désormais comme un facteur décisif pour l'obtention de l'efficacité humaine. En ce qui concerne l'obtention de la performance dans une organisation, Michel S. et Ledru M. (1991) [10] expliquent que, si les compétences sont toujours une source de performance, elles ne suffisent pas à elles seules à en déterminer le niveau. En effet, bien qu'il y ait des personnes pouvant avoir la même compétence, leurs performances par contre peuvent être différentes. Cela veut dire que si la compétence est un savoir-faire, la performance présuppose toujours l'existence d'un vouloir-faire ou motivation, qui vont conduire le salarié à s'engager effectivement dans l'action qui lui est proposée par l'entreprise.

En présence de plusieurs phénomènes, tels que l'hyper compétition, les risques de pénurie de main d'œuvre, et l'accroissement de l'innovation, la performance des organisations repose de plus en plus sur l'excellence individuelle de personnes-clés, personnes que désigne le qualificatif de «Talents ». Autrement dit, l'apparition de cette nouvelle notion fait référence à la guerre des talents que ce soit entre les organisations, ou bien encore entre les pays. Elle est née dans un contexte d'«hyper compétition ", caractérisé par la pénurie de la main d'œuvre qualifiée, l'instabilité et l'incertitude. D'après Mirallès $(2007, p .14)$ « ces phénomènes créent des conditions qui tendent à renforcer les exigences de compétence et d'implication des salariés».

Il faut dire que la mondialisation est à l'origine du phénomène d'hyper compétition entre firmes $(\mathrm{R}$. D'Aveni, 1995) [11]. Mirallès souligne que « L'hyper compétition, c'est avant tout un contexte au sein duquel aucun acteur ne peut prétendre à bénéficier d'un avantage concurrentiel unique et durable (comme par exemple le coût de production ou les barrières à l'entrée), mais dans lequel les différents compétiteurs recherchent des combinaisons éphémères d'avantages concurrentiels variés tels que le délai de mise en marché, la qualité, la capacité financière, la technologie, etc. Par ailleurs, nos économies se «déforment » dans le sens du renversement de la proportion traditionnelle entre coûts de conception et coûts de production des biens et des services ».

Aujourd'hui, se doter des talents dans nos environnements incertains, devient une nécessité urgente afin que les entreprises puissent faire face aux enjeux d'un environnement complexe. La gestion des talents est aujourd'hui liée à ce qu'on appelle « la guerre des talents », car face à la rareté des talents, la fuite des cerveaux, et en présence des chasseurs de têtes, la concurrence en matière des talents est devenue accrue entre les entreprises. Il convient de dire que la notion de talent est apparue au sein de celles-ci notamment pour répondre aux enjeux de compétitivité qui s'avéraient contraignants. C'est pourquoi les entreprises s'orientent de plus en plus vers la mise en place de viviers de talents.

\section{Méthodologie}

Pour identifier les pratiques RH mises en place par les banques marocaines en matière de la gestion des talents, nous avons retenu les nouvelles pratiques de la gestion des talents, à savoir : attirer, accompagner et fidéliser les talents. L'objectif étant de déterminer la place de la gestion des talents au sein des banques marocaines. Pour tenter de répondre à notre problématique, nous avons opté, dans un premier temps, pour une méthodologie de recherche qualitative. Nous avons choisi la méthode de l'étude de cas multiples. En effet, notre échantillon est composé de trois grandes banques marocaines classées premières à l'échelle nationale, à savoir : Attijariwafa bank (AWB), la Banque Populaire (BP), et la Banque Marocaine de Commerce Extérieur (BMCE Bank). Pour le recueil des données, nous avons fait recours à l'observation directe du milieu bancaire, et des différentes sources documentaires. Ensuite, nous avons mobilisé l'outil de l'entretien individuel semi-directif, de face à face, et téléphonique, avec guide d'entretien. 
Dans cette première étape de notre recherche, notre enquête a porté sur un échantillon de 60 personnes représentatives de la population des cadres et des employés de banque (expérimentés et nouvelles recrues) occupant des postes différents au sein de la banque. Pour cela, 45 agences bancaires, filiales des trois banques ont été choisies. Ces agences se situent dans des régions et des villes différentes (des grandes, des moyennes et des petites villes, voire même des patelins).

A ce stade, notre objectif consiste à repérer la place de la gestion des talents au sein de ces banques, en identifiant leurs pratiques RH. Tout d'abord, nous avons mis l'accent sur les nouvelles pratiques RH apparues suite à l'avènement de la notion «Talents ». Ensuite, nous avons analysé les pratiques adoptées par les banques marocaines, afin de voir si cette nouvelle approche est présente ou non dans leur politique RH.

Dans une deuxième étape, nous envisageons de mener une recherche de type quantitatif, tout en exploitant les données récoltées de cette première étude pour approfondir certaines questions émi0ses à l'issue de nos interventions. Il serait donc utile de recourir à la méthodologie quantitative pour appréhender notre problématique différemment en tenant compte d'autres critères quantitatifs, ainsi que de l'analyser en profondeur.

\section{Résultats de la recherche}

Nous allons synthétiser les principaux résultats obtenus dans notre travail de recherche dans le tableau suivant :

Tableau 1. Synthèse des résultats obtenus

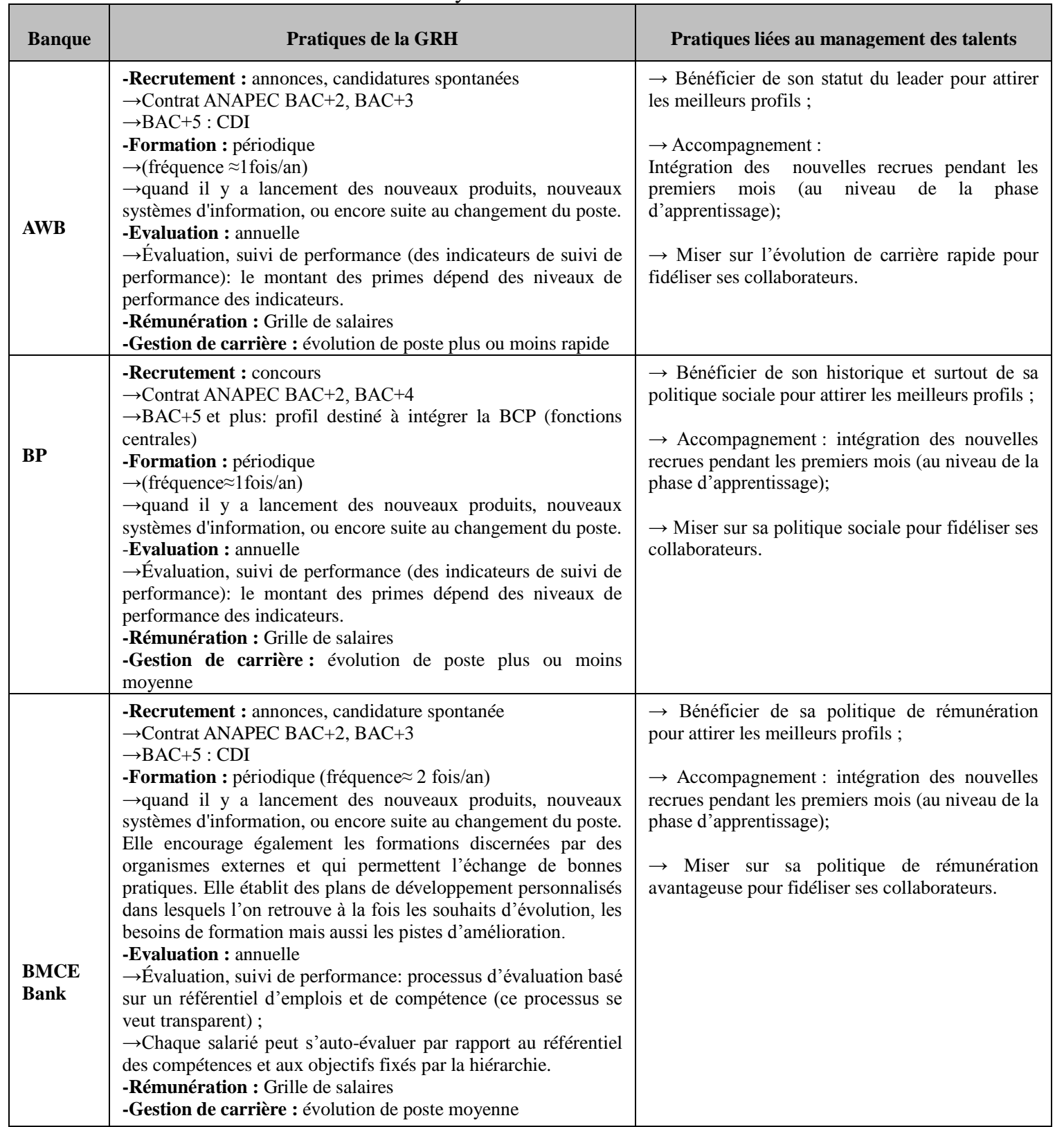

Source : Elaboré par l'auteur sur la base des résultats obtenus 


\section{Discussion des résultats}

Il ressort des données récoltées de notre étude empirique, que les trois banques étudiées ont beaucoup de points de convergence en ce qui concerne leur politique RH. Les résultats de notre recherche ont montré que le modèle classique de la GRH domine encore les pratiques RH des trois banques susvisées. En effet, leurs pratiques RH détectées tournent généralement autour du recrutement, de la rémunération, de la formation, de l'évaluation, et de la gestion des carrières. De même, en analysant la colonne du tableau réservée aux pratiques du management des talents, on remarque que ces nouvelles pratiques sont mises en œuvre seulement en partie par ces trois banques. Par conséquent, on peut dire que la nouvelle approche RH « Talents » a du mal à se faire une place au sein des banques marocaines.

Par ailleurs, nous constatons quelques points de divergences entre les trois cas analysés. Si on prend la politique de fidélisation des salariés par exemple, la BP met l'accent sur sa politique sociale afin de préserver ses salariés, contrairement à AWB dont sa politique de fidélisation repose sur les perspectives d'évolution de carrière. Quant à la BMCE Bank, la rétention de ses salariés est liée principalement à sa politique de rémunération avantageuse.

Nous avons aussi constaté lors de notre étude empirique, qu'il existe deux catégories de talents :

Des talents innovants ;

Des talents performants.

Pour ce qui est des talents innovants, ce sont des personnes dont leur talent se traduit par la production des nouvelles idées, des nouveaux concepts, des nouvelles façons de faire, ... Leur innovation va au-delà du simple travail bien fait, il s'agit de se laisser aller en introduisant quelque chose de nouveau en matière de leur travail. Ces talents innovants sont appréciés pour la créativité et l'innovation qu'ils apportent à leur structure, et qui bien évidement contribuent à son développement et à sa performance. Néanmoins, il reste généralement difficile de mesurer d'une manière quantitative.

Quant aux talents performants, ce sont des personnes qui parviennent à aboutir à des résultats exceptionnels. Dans notre cas, ce type de talent on le trouve surtout chez les personnes dotées d'une agressivité commerciale remarquable, leur grand atout se fait remarquer dans la réalisation des chiffres. A cet effet, les banques mesurent la performance de ces talents en faisant recours aux données fournies par les reportings, et les tableaux de bord permettant de mesurer l'effort fourni par chacun. Pour faire face à la concurrence, les banques aujourd'hui sont beaucoup plus à la recherche de ce type de profils qui leur permettent d'accroitre leurs chiffres d'affaires, d'augmenter leurs résultats, et par conséquence atteindre leur performance.

Notre étude empirique nous amène également à l'idée que les banques marocaines doivent être incitées à détecter les talents internes. En effet, les talents ne se limitent pas à la seule catégorie des cadres. Les talents sont également à puiser dans un vivier plus traditionnel, à savoir les employés de banque.

Les résultats obtenus à la lumière de notre travail de recherche montrent que le but ultime des banques étant l'amélioration de la performance. Pour cette raison, elles sont amenées à revoir leur politique RH. Il s'agit d'introduire un nouveau modèle de management qui tient compte des nouvelles pratiques de GRH. Cependant, nous n'avons pas l'intention à travers notre étude de laisser croire qu'il existe de «meilleures pratiques RH », étant donné que les approches RH sont constamment en évolution et mises à jour. En effet, Peretti (2012) [12], explique qu'il n'y a pas de pratiques universelles en matière de GRH. Celles-ci peuvent être considérées comme performantes lorsqu'elles sont adaptées au contexte, afin de répondre aux défis auxquels l'entreprise est confrontée. Néanmoins, il existe de «bonnes pratiques RH» qui permettent à l'entreprise d'améliorer la manière dont elle travaille, en contribuant à sa performance. Notre objectif étant de montrer les avantages et l'intérêt de cette nouvelle approche RH sur la performance de la banque.

\section{Conclusion}

In fine, il convient de dire que les banques marocaines disposent d'un ensemble d'ingrédients et des atouts en termes de moyens humains, financiers, et organisationnels qui leurs permettent de s'inscrire dans la dynamique du développement de la GRH, en favorisant la mise en place d'une nouvelle approche RH à savoir la gestion des talents. Or, pour ce faire, il ne suffit pas seulement d'attirer et de fidéliser les talents, les banques sont amenées à accompagner ces talents en leur offrant des perspectives intéressantes en matière de carrière. Elles doivent également veiller au bien-être de leurs talents, et développer en permanence leur politique RH. Ainsi, il est peut-être temps que les banques marocaines revoient leurs pratiques RH pour les adapter au contexte actuel. Pour cela, il faut avoir la volonté d'agir et l'esprit d'oser.

Notre recherche comme tout travail de recherche, laisse apparaître des points à améliorer et des limites ouvrant la voie à des futurs champs de recherche. Il nous parait donc judicieux de considérer avec précaution les conclusions exposées et les résultats obtenus d'après notre travail de recherche. En effet, notre échantillon de recherche choisi est caractérisé par un nombre d'observations relativement faible (limité à trois banques). Il n'est pas très représentatif, ce qui réduit la possibilité de généralisation des résultats obtenus. 
Par ailleurs, nous avons rencontré des difficultés liées à l'accès aux informations, et aux documents internes, vu la confidentialité du métier basé sur le fait de ne pas divulguer le secret professionnel, ce qui exige une discrétion absolue.

Néanmoins, nous estimons que ces résultats apportent une valeur ajoutée, dans la mesure où, d'une part, ce travail de recherche nous a permis de porter une réflexion du début jusqu'à la fin sur la place du talent au sein de la banque marocaine. Et d'autre part, ils nous amènent à comprendre l'enjeu actuel des banques marocaines, qui affrontent de nombreux défis économiques, technologiques, et sociaux. Autrement dit, il apporte un éclairage sur cette nouvelle approche RH qui constitue un changement important dans les pratiques de GRH.

Cela constitue certes une base à fort potentiel pour la mise en ouvre de la seconde partie de notre recherche, à savoir une enquête quantitative sur la même problématique de recherche, mais basée sur un échantillon plus représentatif des banques marocaines. Aussi, pour élargir notre champ d'analyse, il serait intéressant de traiter le cas des banques marocaines filiales des banques étrangères notamment françaises, comme la BMCI (Banque Marocaine pour le Commerce et l'Industrie), filiale de la banque française BNP Paribas, ou Crédit du Maroc, filiale de la banque française Crédit Agricole, ou bien encore la Société Générale, filiale de la banque française Société Générale. L'objectif étant d'analyser les pratiques RH mises en œuvre par ces banques, pour voir si elles appliquent la même politique RH de leur banque mère, c'est-à-dire la même que celle appliquée en France, ou bien elles appliquent plutôt une politique RH adaptée au contexte marocain, en tenant compte des spécificités de ce pays en voie de développement.

\section{Références}

[1] L. Cadin, La gestion des talents au regard de l'évolution des carrières : Proposition de cartographie des courants de la gestion des talents, Carriérologie, 13(1-2), 2013, 15-29.

[2] M. Thévenet et C. Dejoux, La gestion des talents: La GRH d'après-crise (Dunod, Paris, 2010)

[3] E. Michaels, H.Handfield-Jones, and al., The war for talent, Boston: Harvard Business School Press, 2001.

[4] R. E. Lewis and R. J. Heckman, Talent management: A critical review, Human Resource Management Review, 2006, 139154.

[5] P. Mirallès, La gestion des talents : émergence d'un nouveau modèle de management ?, Management \& Avenir 1(11), 2007, 29-42.

[6] B. Chaminade, Identifiez et fidélisez vos salariés de talent (Afnor, 2003).

[7] F. De Bry et C. Gourdon, Talent soutenable et entreprise, In J.M. Peretti (dir), Tous Talentueux Développer Les Talents et Les Potentiels Dans L'entreprise, (Paris, 2009, p.59).

[8] H. Bouquin. Le contrôle de gestion (Presses Universitaires de France, 1986)

[9] A. Bourguignon, Peut-on définir la performance? Revue Française de Comptabilité, 1995, 61-66.

[10] S. Michel et M. Ledru, Capital-Compétence dans l'entreprise (ESF éditeur, 1991).

[11] R. D’Aveni (1995). Hypercompétition (Vuibert, Paris, 1995).

[12] J.M. PERETTI, Gestion des Ressources Humaines (Vuibert, Paris, 2012). 